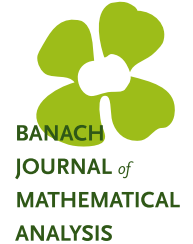

Banach J. Math. Anal. 10 (2016), no. 2, 430-450

http://dx.doi.org/10.1215/17358787-3589397

ISSN: $1735-8787$ (electronic)

http://projecteuclid.org/bjma

\title{
VECTOR-VALUED HILBERT TRANSFORMS ALONG CURVES
}

\author{
GUIXIANG HONG ${ }^{1}$ and HONGHAI LIU ${ }^{2 *}$ \\ Communicated by Q. Xu
}

\begin{abstract}
In this paper, we show that Hilbert transforms along some curves are bounded on $L^{p}\left(\mathbb{R}^{n} ; X\right)$ for some $1<p<\infty$ and some UMD spaces $X$. In particular, we prove that Hilbert transforms along some curves are completely $L^{p}$-bounded in the terminology from operator space theory. Moreover, we obtain the $L^{p}\left(\mathbb{R}^{n} ; X\right)$-boundedness of anisotropic singular integrals by using the "method of rotations" of Calderón-Zygmund. All these results extend preexisting related ones.
\end{abstract}

\section{INTRODUCTION}

The question of whether the mapping properties of singular integral operators could be extended to the Lebesgue-Bôhner spaces $L^{p}\left(\mathbb{R}^{n} ; X\right)(1<p<\infty)$ of vector-valued functions was taken up by several authors in the 60s. In [1], Benedek, Calderón, and Panzone observed that the boundedness on $L^{p_{0}}\left(\mathbb{R}^{n} ; X\right)$ for one $1<p_{0}<\infty$ of a singular integral operator, together with Hörmander's condition, implies its boundedness on $L^{p}\left(\mathbb{R}^{n} ; X\right)$ for all $1<p<\infty$. However, to actually get the $L^{p_{0}}\left(\mathbb{R}^{n} ; X\right)$-boundedness (something that was immediate for $p_{0}=2$ in the scalar-valued) turned out to be a significantly difficult task except in the case $X=L^{p_{0}}(\Omega)$ for some measure space $\Omega$.

The first progress made in this direction is Burkholder's [3] extension of Riesz's classical theorem on the $L^{p}$-boundedness of the Hilbert transform, where it was shown that if the underlying Banach space $X$ satisfies the so-called UMD-property,

Copyright 2016 by the Tusi Mathematical Research Group.

Received Apr. 9, 2015; Accepted Jul. 27, 2015.

${ }^{*}$ Corresponding author.

2010 Mathematics Subject Classification. Primary 43A32; Secondary 46 B99.

Keywords. Hilbert transforms along curves, weighted Hörmander condition, UMD spaces, completely bounded, analytic interpolation. 




Banach J. Math. Anal. 10 (2016), no. 2, 430-450

http://dx.doi.org/10.1215/17358787-3589397

ISSN: $1735-8787$ (electronic)

http://projecteuclid.org/bjma

\title{
VECTOR-VALUED HILBERT TRANSFORMS ALONG CURVES
}

\author{
GUIXIANG HONG ${ }^{1}$ and HONGHAI LIU ${ }^{2 *}$ \\ Communicated by Q. Xu
}

\begin{abstract}
In this paper, we show that Hilbert transforms along some curves are bounded on $L^{p}\left(\mathbb{R}^{n} ; X\right)$ for some $1<p<\infty$ and some UMD spaces $X$. In particular, we prove that Hilbert transforms along some curves are completely $L^{p}$-bounded in the terminology from operator space theory. Moreover, we obtain the $L^{p}\left(\mathbb{R}^{n} ; X\right)$-boundedness of anisotropic singular integrals by using the "method of rotations" of Calderón-Zygmund. All these results extend preexisting related ones.
\end{abstract}

\section{INTRODUCTION}

The question of whether the mapping properties of singular integral operators could be extended to the Lebesgue-Bôhner spaces $L^{p}\left(\mathbb{R}^{n} ; X\right)(1<p<\infty)$ of vector-valued functions was taken up by several authors in the 60s. In [1], Benedek, Calderón, and Panzone observed that the boundedness on $L^{p_{0}}\left(\mathbb{R}^{n} ; X\right)$ for one $1<p_{0}<\infty$ of a singular integral operator, together with Hörmander's condition, implies its boundedness on $L^{p}\left(\mathbb{R}^{n} ; X\right)$ for all $1<p<\infty$. However, to actually get the $L^{p_{0}}\left(\mathbb{R}^{n} ; X\right)$-boundedness (something that was immediate for $p_{0}=2$ in the scalar-valued) turned out to be a significantly difficult task except in the case $X=L^{p_{0}}(\Omega)$ for some measure space $\Omega$.

The first progress made in this direction is Burkholder's [3] extension of Riesz's classical theorem on the $L^{p}$-boundedness of the Hilbert transform, where it was shown that if the underlying Banach space $X$ satisfies the so-called UMD-property,

Copyright 2016 by the Tusi Mathematical Research Group.

Received Apr. 9, 2015; Accepted Jul. 27, 2015.

${ }^{*}$ Corresponding author.

2010 Mathematics Subject Classification. Primary 43A32; Secondary 46 B99.

Keywords. Hilbert transforms along curves, weighted Hörmander condition, UMD spaces, completely bounded, analytic interpolation. 


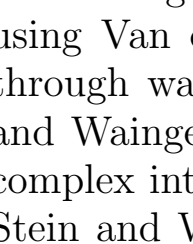

Banach J. Math. Anal. 10 (2016), no. 2, 430-450

http://dx.doi.org/10.1215/17358787-3589397

ISSN: $1735-8787$ (electronic)

http://projecteuclid.org/bjma

\title{
VECTOR-VALUED HILBERT TRANSFORMS ALONG CURVES
}

\author{
GUIXIANG HONG ${ }^{1}$ and HONGHAI LIU ${ }^{2 *}$ \\ Communicated by Q. Xu
}

\begin{abstract}
In this paper, we show that Hilbert transforms along some curves are bounded on $L^{p}\left(\mathbb{R}^{n} ; X\right)$ for some $1<p<\infty$ and some UMD spaces $X$. In particular, we prove that Hilbert transforms along some curves are completely $L^{p}$-bounded in the terminology from operator space theory. Moreover, we obtain the $L^{p}\left(\mathbb{R}^{n} ; X\right)$-boundedness of anisotropic singular integrals by using the "method of rotations" of Calderón-Zygmund. All these results extend preexisting related ones.
\end{abstract}

\section{INTRODUCTION}

The question of whether the mapping properties of singular integral operators could be extended to the Lebesgue-Bôhner spaces $L^{p}\left(\mathbb{R}^{n} ; X\right)(1<p<\infty)$ of vector-valued functions was taken up by several authors in the 60s. In [1], Benedek, Calderón, and Panzone observed that the boundedness on $L^{p_{0}}\left(\mathbb{R}^{n} ; X\right)$ for one $1<p_{0}<\infty$ of a singular integral operator, together with Hörmander's condition, implies its boundedness on $L^{p}\left(\mathbb{R}^{n} ; X\right)$ for all $1<p<\infty$. However, to actually get the $L^{p_{0}}\left(\mathbb{R}^{n} ; X\right)$-boundedness (something that was immediate for $p_{0}=2$ in the scalar-valued) turned out to be a significantly difficult task except in the case $X=L^{p_{0}}(\Omega)$ for some measure space $\Omega$.

The first progress made in this direction is Burkholder's [3] extension of Riesz's classical theorem on the $L^{p}$-boundedness of the Hilbert transform, where it was shown that if the underlying Banach space $X$ satisfies the so-called UMD-property,

Copyright 2016 by the Tusi Mathematical Research Group.

Received Apr. 9, 2015; Accepted Jul. 27, 2015.

${ }^{*}$ Corresponding author.

2010 Mathematics Subject Classification. Primary 43A32; Secondary 46 B99.

Keywords. Hilbert transforms along curves, weighted Hörmander condition, UMD spaces, completely bounded, analytic interpolation. 


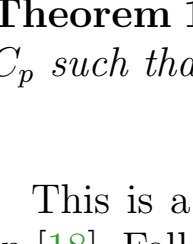

Banach J. Math. Anal. 10 (2016), no. 2, 430-450

http://dx.doi.org/10.1215/17358787-3589397

ISSN: $1735-8787$ (electronic)

http://projecteuclid.org/bjma

\title{
VECTOR-VALUED HILBERT TRANSFORMS ALONG CURVES
}

\author{
GUIXIANG HONG ${ }^{1}$ and HONGHAI LIU ${ }^{2 *}$ \\ Communicated by Q. Xu
}

\begin{abstract}
In this paper, we show that Hilbert transforms along some curves are bounded on $L^{p}\left(\mathbb{R}^{n} ; X\right)$ for some $1<p<\infty$ and some UMD spaces $X$. In particular, we prove that Hilbert transforms along some curves are completely $L^{p}$-bounded in the terminology from operator space theory. Moreover, we obtain the $L^{p}\left(\mathbb{R}^{n} ; X\right)$-boundedness of anisotropic singular integrals by using the "method of rotations" of Calderón-Zygmund. All these results extend preexisting related ones.
\end{abstract}

\section{INTRODUCTION}

The question of whether the mapping properties of singular integral operators could be extended to the Lebesgue-Bôhner spaces $L^{p}\left(\mathbb{R}^{n} ; X\right)(1<p<\infty)$ of vector-valued functions was taken up by several authors in the 60s. In [1], Benedek, Calderón, and Panzone observed that the boundedness on $L^{p_{0}}\left(\mathbb{R}^{n} ; X\right)$ for one $1<p_{0}<\infty$ of a singular integral operator, together with Hörmander's condition, implies its boundedness on $L^{p}\left(\mathbb{R}^{n} ; X\right)$ for all $1<p<\infty$. However, to actually get the $L^{p_{0}}\left(\mathbb{R}^{n} ; X\right)$-boundedness (something that was immediate for $p_{0}=2$ in the scalar-valued) turned out to be a significantly difficult task except in the case $X=L^{p_{0}}(\Omega)$ for some measure space $\Omega$.

The first progress made in this direction is Burkholder's [3] extension of Riesz's classical theorem on the $L^{p}$-boundedness of the Hilbert transform, where it was shown that if the underlying Banach space $X$ satisfies the so-called UMD-property,

Copyright 2016 by the Tusi Mathematical Research Group.

Received Apr. 9, 2015; Accepted Jul. 27, 2015.

${ }^{*}$ Corresponding author.

2010 Mathematics Subject Classification. Primary 43A32; Secondary 46 B99.

Keywords. Hilbert transforms along curves, weighted Hörmander condition, UMD spaces, completely bounded, analytic interpolation. 


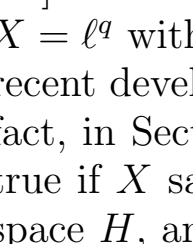

Banach J. Math. Anal. 10 (2016), no. 2, 430-450

http://dx.doi.org/10.1215/17358787-3589397

ISSN: $1735-8787$ (electronic)

http://projecteuclid.org/bjma

\title{
VECTOR-VALUED HILBERT TRANSFORMS ALONG CURVES
}

\author{
GUIXIANG HONG ${ }^{1}$ and HONGHAI LIU ${ }^{2 *}$ \\ Communicated by Q. Xu
}

\begin{abstract}
In this paper, we show that Hilbert transforms along some curves are bounded on $L^{p}\left(\mathbb{R}^{n} ; X\right)$ for some $1<p<\infty$ and some UMD spaces $X$. In particular, we prove that Hilbert transforms along some curves are completely $L^{p}$-bounded in the terminology from operator space theory. Moreover, we obtain the $L^{p}\left(\mathbb{R}^{n} ; X\right)$-boundedness of anisotropic singular integrals by using the "method of rotations" of Calderón-Zygmund. All these results extend preexisting related ones.
\end{abstract}

\section{INTRODUCTION}

The question of whether the mapping properties of singular integral operators could be extended to the Lebesgue-Bôhner spaces $L^{p}\left(\mathbb{R}^{n} ; X\right)(1<p<\infty)$ of vector-valued functions was taken up by several authors in the 60s. In [1], Benedek, Calderón, and Panzone observed that the boundedness on $L^{p_{0}}\left(\mathbb{R}^{n} ; X\right)$ for one $1<p_{0}<\infty$ of a singular integral operator, together with Hörmander's condition, implies its boundedness on $L^{p}\left(\mathbb{R}^{n} ; X\right)$ for all $1<p<\infty$. However, to actually get the $L^{p_{0}}\left(\mathbb{R}^{n} ; X\right)$-boundedness (something that was immediate for $p_{0}=2$ in the scalar-valued) turned out to be a significantly difficult task except in the case $X=L^{p_{0}}(\Omega)$ for some measure space $\Omega$.

The first progress made in this direction is Burkholder's [3] extension of Riesz's classical theorem on the $L^{p}$-boundedness of the Hilbert transform, where it was shown that if the underlying Banach space $X$ satisfies the so-called UMD-property,

Copyright 2016 by the Tusi Mathematical Research Group.

Received Apr. 9, 2015; Accepted Jul. 27, 2015.

${ }^{*}$ Corresponding author.

2010 Mathematics Subject Classification. Primary 43A32; Secondary 46 B99.

Keywords. Hilbert transforms along curves, weighted Hörmander condition, UMD spaces, completely bounded, analytic interpolation. 


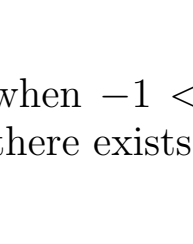

Banach J. Math. Anal. 10 (2016), no. 2, 430-450

http://dx.doi.org/10.1215/17358787-3589397

ISSN: $1735-8787$ (electronic)

http://projecteuclid.org/bjma

\title{
VECTOR-VALUED HILBERT TRANSFORMS ALONG CURVES
}

\author{
GUIXIANG HONG ${ }^{1}$ and HONGHAI LIU ${ }^{2 *}$ \\ Communicated by Q. Xu
}

\begin{abstract}
In this paper, we show that Hilbert transforms along some curves are bounded on $L^{p}\left(\mathbb{R}^{n} ; X\right)$ for some $1<p<\infty$ and some UMD spaces $X$. In particular, we prove that Hilbert transforms along some curves are completely $L^{p}$-bounded in the terminology from operator space theory. Moreover, we obtain the $L^{p}\left(\mathbb{R}^{n} ; X\right)$-boundedness of anisotropic singular integrals by using the "method of rotations" of Calderón-Zygmund. All these results extend preexisting related ones.
\end{abstract}

\section{INTRODUCTION}

The question of whether the mapping properties of singular integral operators could be extended to the Lebesgue-Bôhner spaces $L^{p}\left(\mathbb{R}^{n} ; X\right)(1<p<\infty)$ of vector-valued functions was taken up by several authors in the 60s. In [1], Benedek, Calderón, and Panzone observed that the boundedness on $L^{p_{0}}\left(\mathbb{R}^{n} ; X\right)$ for one $1<p_{0}<\infty$ of a singular integral operator, together with Hörmander's condition, implies its boundedness on $L^{p}\left(\mathbb{R}^{n} ; X\right)$ for all $1<p<\infty$. However, to actually get the $L^{p_{0}}\left(\mathbb{R}^{n} ; X\right)$-boundedness (something that was immediate for $p_{0}=2$ in the scalar-valued) turned out to be a significantly difficult task except in the case $X=L^{p_{0}}(\Omega)$ for some measure space $\Omega$.

The first progress made in this direction is Burkholder's [3] extension of Riesz's classical theorem on the $L^{p}$-boundedness of the Hilbert transform, where it was shown that if the underlying Banach space $X$ satisfies the so-called UMD-property,

Copyright 2016 by the Tusi Mathematical Research Group.

Received Apr. 9, 2015; Accepted Jul. 27, 2015.

${ }^{*}$ Corresponding author.

2010 Mathematics Subject Classification. Primary 43A32; Secondary 46 B99.

Keywords. Hilbert transforms along curves, weighted Hörmander condition, UMD spaces, completely bounded, analytic interpolation. 


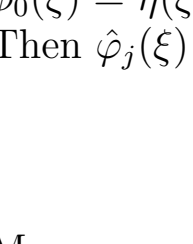

Banach J. Math. Anal. 10 (2016), no. 2, 430-450

http://dx.doi.org/10.1215/17358787-3589397

ISSN: $1735-8787$ (electronic)

http://projecteuclid.org/bjma

\title{
VECTOR-VALUED HILBERT TRANSFORMS ALONG CURVES
}

\author{
GUIXIANG HONG ${ }^{1}$ and HONGHAI LIU ${ }^{2 *}$ \\ Communicated by Q. Xu
}

\begin{abstract}
In this paper, we show that Hilbert transforms along some curves are bounded on $L^{p}\left(\mathbb{R}^{n} ; X\right)$ for some $1<p<\infty$ and some UMD spaces $X$. In particular, we prove that Hilbert transforms along some curves are completely $L^{p}$-bounded in the terminology from operator space theory. Moreover, we obtain the $L^{p}\left(\mathbb{R}^{n} ; X\right)$-boundedness of anisotropic singular integrals by using the "method of rotations" of Calderón-Zygmund. All these results extend preexisting related ones.
\end{abstract}

\section{INTRODUCTION}

The question of whether the mapping properties of singular integral operators could be extended to the Lebesgue-Bôhner spaces $L^{p}\left(\mathbb{R}^{n} ; X\right)(1<p<\infty)$ of vector-valued functions was taken up by several authors in the 60s. In [1], Benedek, Calderón, and Panzone observed that the boundedness on $L^{p_{0}}\left(\mathbb{R}^{n} ; X\right)$ for one $1<p_{0}<\infty$ of a singular integral operator, together with Hörmander's condition, implies its boundedness on $L^{p}\left(\mathbb{R}^{n} ; X\right)$ for all $1<p<\infty$. However, to actually get the $L^{p_{0}}\left(\mathbb{R}^{n} ; X\right)$-boundedness (something that was immediate for $p_{0}=2$ in the scalar-valued) turned out to be a significantly difficult task except in the case $X=L^{p_{0}}(\Omega)$ for some measure space $\Omega$.

The first progress made in this direction is Burkholder's [3] extension of Riesz's classical theorem on the $L^{p}$-boundedness of the Hilbert transform, where it was shown that if the underlying Banach space $X$ satisfies the so-called UMD-property,

Copyright 2016 by the Tusi Mathematical Research Group.

Received Apr. 9, 2015; Accepted Jul. 27, 2015.

${ }^{*}$ Corresponding author.

2010 Mathematics Subject Classification. Primary 43A32; Secondary 46 B99.

Keywords. Hilbert transforms along curves, weighted Hörmander condition, UMD spaces, completely bounded, analytic interpolation. 




Banach J. Math. Anal. 10 (2016), no. 2, 430-450

http://dx.doi.org/10.1215/17358787-3589397

ISSN: $1735-8787$ (electronic)

http://projecteuclid.org/bjma

\title{
VECTOR-VALUED HILBERT TRANSFORMS ALONG CURVES
}

\author{
GUIXIANG HONG ${ }^{1}$ and HONGHAI LIU ${ }^{2 *}$ \\ Communicated by Q. Xu
}

\begin{abstract}
In this paper, we show that Hilbert transforms along some curves are bounded on $L^{p}\left(\mathbb{R}^{n} ; X\right)$ for some $1<p<\infty$ and some UMD spaces $X$. In particular, we prove that Hilbert transforms along some curves are completely $L^{p}$-bounded in the terminology from operator space theory. Moreover, we obtain the $L^{p}\left(\mathbb{R}^{n} ; X\right)$-boundedness of anisotropic singular integrals by using the "method of rotations" of Calderón-Zygmund. All these results extend preexisting related ones.
\end{abstract}

\section{INTRODUCTION}

The question of whether the mapping properties of singular integral operators could be extended to the Lebesgue-Bôhner spaces $L^{p}\left(\mathbb{R}^{n} ; X\right)(1<p<\infty)$ of vector-valued functions was taken up by several authors in the 60s. In [1], Benedek, Calderón, and Panzone observed that the boundedness on $L^{p_{0}}\left(\mathbb{R}^{n} ; X\right)$ for one $1<p_{0}<\infty$ of a singular integral operator, together with Hörmander's condition, implies its boundedness on $L^{p}\left(\mathbb{R}^{n} ; X\right)$ for all $1<p<\infty$. However, to actually get the $L^{p_{0}}\left(\mathbb{R}^{n} ; X\right)$-boundedness (something that was immediate for $p_{0}=2$ in the scalar-valued) turned out to be a significantly difficult task except in the case $X=L^{p_{0}}(\Omega)$ for some measure space $\Omega$.

The first progress made in this direction is Burkholder's [3] extension of Riesz's classical theorem on the $L^{p}$-boundedness of the Hilbert transform, where it was shown that if the underlying Banach space $X$ satisfies the so-called UMD-property,

Copyright 2016 by the Tusi Mathematical Research Group.

Received Apr. 9, 2015; Accepted Jul. 27, 2015.

${ }^{*}$ Corresponding author.

2010 Mathematics Subject Classification. Primary 43A32; Secondary 46 B99.

Keywords. Hilbert transforms along curves, weighted Hörmander condition, UMD spaces, completely bounded, analytic interpolation. 


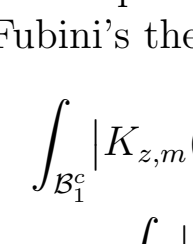

Banach J. Math. Anal. 10 (2016), no. 2, 430-450

http://dx.doi.org/10.1215/17358787-3589397

ISSN: $1735-8787$ (electronic)

http://projecteuclid.org/bjma

\title{
VECTOR-VALUED HILBERT TRANSFORMS ALONG CURVES
}

\author{
GUIXIANG HONG ${ }^{1}$ and HONGHAI LIU ${ }^{2 *}$ \\ Communicated by Q. Xu
}

\begin{abstract}
In this paper, we show that Hilbert transforms along some curves are bounded on $L^{p}\left(\mathbb{R}^{n} ; X\right)$ for some $1<p<\infty$ and some UMD spaces $X$. In particular, we prove that Hilbert transforms along some curves are completely $L^{p}$-bounded in the terminology from operator space theory. Moreover, we obtain the $L^{p}\left(\mathbb{R}^{n} ; X\right)$-boundedness of anisotropic singular integrals by using the "method of rotations" of Calderón-Zygmund. All these results extend preexisting related ones.
\end{abstract}

\section{INTRODUCTION}

The question of whether the mapping properties of singular integral operators could be extended to the Lebesgue-Bôhner spaces $L^{p}\left(\mathbb{R}^{n} ; X\right)(1<p<\infty)$ of vector-valued functions was taken up by several authors in the 60s. In [1], Benedek, Calderón, and Panzone observed that the boundedness on $L^{p_{0}}\left(\mathbb{R}^{n} ; X\right)$ for one $1<p_{0}<\infty$ of a singular integral operator, together with Hörmander's condition, implies its boundedness on $L^{p}\left(\mathbb{R}^{n} ; X\right)$ for all $1<p<\infty$. However, to actually get the $L^{p_{0}}\left(\mathbb{R}^{n} ; X\right)$-boundedness (something that was immediate for $p_{0}=2$ in the scalar-valued) turned out to be a significantly difficult task except in the case $X=L^{p_{0}}(\Omega)$ for some measure space $\Omega$.

The first progress made in this direction is Burkholder's [3] extension of Riesz's classical theorem on the $L^{p}$-boundedness of the Hilbert transform, where it was shown that if the underlying Banach space $X$ satisfies the so-called UMD-property,

Copyright 2016 by the Tusi Mathematical Research Group.

Received Apr. 9, 2015; Accepted Jul. 27, 2015.

${ }^{*}$ Corresponding author.

2010 Mathematics Subject Classification. Primary 43A32; Secondary 46 B99.

Keywords. Hilbert transforms along curves, weighted Hörmander condition, UMD spaces, completely bounded, analytic interpolation. 


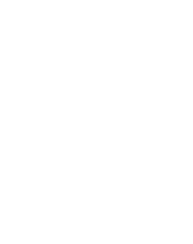

Banach J. Math. Anal. 10 (2016), no. 2, 430-450

http://dx.doi.org/10.1215/17358787-3589397

ISSN: $1735-8787$ (electronic)

http://projecteuclid.org/bjma

\title{
VECTOR-VALUED HILBERT TRANSFORMS ALONG CURVES
}

\author{
GUIXIANG HONG ${ }^{1}$ and HONGHAI LIU ${ }^{2 *}$ \\ Communicated by Q. Xu
}

\begin{abstract}
In this paper, we show that Hilbert transforms along some curves are bounded on $L^{p}\left(\mathbb{R}^{n} ; X\right)$ for some $1<p<\infty$ and some UMD spaces $X$. In particular, we prove that Hilbert transforms along some curves are completely $L^{p}$-bounded in the terminology from operator space theory. Moreover, we obtain the $L^{p}\left(\mathbb{R}^{n} ; X\right)$-boundedness of anisotropic singular integrals by using the "method of rotations" of Calderón-Zygmund. All these results extend preexisting related ones.
\end{abstract}

\section{INTRODUCTION}

The question of whether the mapping properties of singular integral operators could be extended to the Lebesgue-Bôhner spaces $L^{p}\left(\mathbb{R}^{n} ; X\right)(1<p<\infty)$ of vector-valued functions was taken up by several authors in the 60s. In [1], Benedek, Calderón, and Panzone observed that the boundedness on $L^{p_{0}}\left(\mathbb{R}^{n} ; X\right)$ for one $1<p_{0}<\infty$ of a singular integral operator, together with Hörmander's condition, implies its boundedness on $L^{p}\left(\mathbb{R}^{n} ; X\right)$ for all $1<p<\infty$. However, to actually get the $L^{p_{0}}\left(\mathbb{R}^{n} ; X\right)$-boundedness (something that was immediate for $p_{0}=2$ in the scalar-valued) turned out to be a significantly difficult task except in the case $X=L^{p_{0}}(\Omega)$ for some measure space $\Omega$.

The first progress made in this direction is Burkholder's [3] extension of Riesz's classical theorem on the $L^{p}$-boundedness of the Hilbert transform, where it was shown that if the underlying Banach space $X$ satisfies the so-called UMD-property,

Copyright 2016 by the Tusi Mathematical Research Group.

Received Apr. 9, 2015; Accepted Jul. 27, 2015.

${ }^{*}$ Corresponding author.

2010 Mathematics Subject Classification. Primary 43A32; Secondary 46 B99.

Keywords. Hilbert transforms along curves, weighted Hörmander condition, UMD spaces, completely bounded, analytic interpolation. 


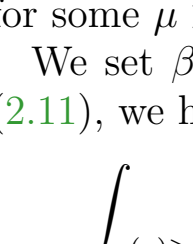

Banach J. Math. Anal. 10 (2016), no. 2, 430-450

http://dx.doi.org/10.1215/17358787-3589397

ISSN: $1735-8787$ (electronic)

http://projecteuclid.org/bjma

\title{
VECTOR-VALUED HILBERT TRANSFORMS ALONG CURVES
}

\author{
GUIXIANG HONG ${ }^{1}$ and HONGHAI LIU ${ }^{2 *}$ \\ Communicated by Q. Xu
}

\begin{abstract}
In this paper, we show that Hilbert transforms along some curves are bounded on $L^{p}\left(\mathbb{R}^{n} ; X\right)$ for some $1<p<\infty$ and some UMD spaces $X$. In particular, we prove that Hilbert transforms along some curves are completely $L^{p}$-bounded in the terminology from operator space theory. Moreover, we obtain the $L^{p}\left(\mathbb{R}^{n} ; X\right)$-boundedness of anisotropic singular integrals by using the "method of rotations" of Calderón-Zygmund. All these results extend preexisting related ones.
\end{abstract}

\section{INTRODUCTION}

The question of whether the mapping properties of singular integral operators could be extended to the Lebesgue-Bôhner spaces $L^{p}\left(\mathbb{R}^{n} ; X\right)(1<p<\infty)$ of vector-valued functions was taken up by several authors in the 60s. In [1], Benedek, Calderón, and Panzone observed that the boundedness on $L^{p_{0}}\left(\mathbb{R}^{n} ; X\right)$ for one $1<p_{0}<\infty$ of a singular integral operator, together with Hörmander's condition, implies its boundedness on $L^{p}\left(\mathbb{R}^{n} ; X\right)$ for all $1<p<\infty$. However, to actually get the $L^{p_{0}}\left(\mathbb{R}^{n} ; X\right)$-boundedness (something that was immediate for $p_{0}=2$ in the scalar-valued) turned out to be a significantly difficult task except in the case $X=L^{p_{0}}(\Omega)$ for some measure space $\Omega$.

The first progress made in this direction is Burkholder's [3] extension of Riesz's classical theorem on the $L^{p}$-boundedness of the Hilbert transform, where it was shown that if the underlying Banach space $X$ satisfies the so-called UMD-property,

Copyright 2016 by the Tusi Mathematical Research Group.

Received Apr. 9, 2015; Accepted Jul. 27, 2015.

${ }^{*}$ Corresponding author.

2010 Mathematics Subject Classification. Primary 43A32; Secondary 46 B99.

Keywords. Hilbert transforms along curves, weighted Hörmander condition, UMD spaces, completely bounded, analytic interpolation. 


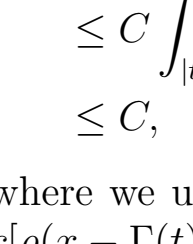

Banach J. Math. Anal. 10 (2016), no. 2, 430-450

http://dx.doi.org/10.1215/17358787-3589397

ISSN: $1735-8787$ (electronic)

http://projecteuclid.org/bjma

\title{
VECTOR-VALUED HILBERT TRANSFORMS ALONG CURVES
}

\author{
GUIXIANG HONG ${ }^{1}$ and HONGHAI LIU ${ }^{2 *}$ \\ Communicated by Q. Xu
}

\begin{abstract}
In this paper, we show that Hilbert transforms along some curves are bounded on $L^{p}\left(\mathbb{R}^{n} ; X\right)$ for some $1<p<\infty$ and some UMD spaces $X$. In particular, we prove that Hilbert transforms along some curves are completely $L^{p}$-bounded in the terminology from operator space theory. Moreover, we obtain the $L^{p}\left(\mathbb{R}^{n} ; X\right)$-boundedness of anisotropic singular integrals by using the "method of rotations" of Calderón-Zygmund. All these results extend preexisting related ones.
\end{abstract}

\section{INTRODUCTION}

The question of whether the mapping properties of singular integral operators could be extended to the Lebesgue-Bôhner spaces $L^{p}\left(\mathbb{R}^{n} ; X\right)(1<p<\infty)$ of vector-valued functions was taken up by several authors in the 60s. In [1], Benedek, Calderón, and Panzone observed that the boundedness on $L^{p_{0}}\left(\mathbb{R}^{n} ; X\right)$ for one $1<p_{0}<\infty$ of a singular integral operator, together with Hörmander's condition, implies its boundedness on $L^{p}\left(\mathbb{R}^{n} ; X\right)$ for all $1<p<\infty$. However, to actually get the $L^{p_{0}}\left(\mathbb{R}^{n} ; X\right)$-boundedness (something that was immediate for $p_{0}=2$ in the scalar-valued) turned out to be a significantly difficult task except in the case $X=L^{p_{0}}(\Omega)$ for some measure space $\Omega$.

The first progress made in this direction is Burkholder's [3] extension of Riesz's classical theorem on the $L^{p}$-boundedness of the Hilbert transform, where it was shown that if the underlying Banach space $X$ satisfies the so-called UMD-property,

Copyright 2016 by the Tusi Mathematical Research Group.

Received Apr. 9, 2015; Accepted Jul. 27, 2015.

${ }^{*}$ Corresponding author.

2010 Mathematics Subject Classification. Primary 43A32; Secondary 46 B99.

Keywords. Hilbert transforms along curves, weighted Hörmander condition, UMD spaces, completely bounded, analytic interpolation. 


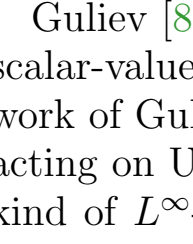

Banach J. Math. Anal. 10 (2016), no. 2, 430-450

http://dx.doi.org/10.1215/17358787-3589397

ISSN: $1735-8787$ (electronic)

http://projecteuclid.org/bjma

\title{
VECTOR-VALUED HILBERT TRANSFORMS ALONG CURVES
}

\author{
GUIXIANG HONG ${ }^{1}$ and HONGHAI LIU ${ }^{2 *}$ \\ Communicated by Q. Xu
}

\begin{abstract}
In this paper, we show that Hilbert transforms along some curves are bounded on $L^{p}\left(\mathbb{R}^{n} ; X\right)$ for some $1<p<\infty$ and some UMD spaces $X$. In particular, we prove that Hilbert transforms along some curves are completely $L^{p}$-bounded in the terminology from operator space theory. Moreover, we obtain the $L^{p}\left(\mathbb{R}^{n} ; X\right)$-boundedness of anisotropic singular integrals by using the "method of rotations" of Calderón-Zygmund. All these results extend preexisting related ones.
\end{abstract}

\section{INTRODUCTION}

The question of whether the mapping properties of singular integral operators could be extended to the Lebesgue-Bôhner spaces $L^{p}\left(\mathbb{R}^{n} ; X\right)(1<p<\infty)$ of vector-valued functions was taken up by several authors in the 60s. In [1], Benedek, Calderón, and Panzone observed that the boundedness on $L^{p_{0}}\left(\mathbb{R}^{n} ; X\right)$ for one $1<p_{0}<\infty$ of a singular integral operator, together with Hörmander's condition, implies its boundedness on $L^{p}\left(\mathbb{R}^{n} ; X\right)$ for all $1<p<\infty$. However, to actually get the $L^{p_{0}}\left(\mathbb{R}^{n} ; X\right)$-boundedness (something that was immediate for $p_{0}=2$ in the scalar-valued) turned out to be a significantly difficult task except in the case $X=L^{p_{0}}(\Omega)$ for some measure space $\Omega$.

The first progress made in this direction is Burkholder's [3] extension of Riesz's classical theorem on the $L^{p}$-boundedness of the Hilbert transform, where it was shown that if the underlying Banach space $X$ satisfies the so-called UMD-property,

Copyright 2016 by the Tusi Mathematical Research Group.

Received Apr. 9, 2015; Accepted Jul. 27, 2015.

${ }^{*}$ Corresponding author.

2010 Mathematics Subject Classification. Primary 43A32; Secondary 46 B99.

Keywords. Hilbert transforms along curves, weighted Hörmander condition, UMD spaces, completely bounded, analytic interpolation. 


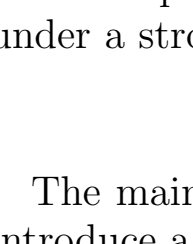

Banach J. Math. Anal. 10 (2016), no. 2, 430-450

http://dx.doi.org/10.1215/17358787-3589397

ISSN: $1735-8787$ (electronic)

http://projecteuclid.org/bjma

\title{
VECTOR-VALUED HILBERT TRANSFORMS ALONG CURVES
}

\author{
GUIXIANG HONG ${ }^{1}$ and HONGHAI LIU ${ }^{2 *}$ \\ Communicated by Q. Xu
}

\begin{abstract}
In this paper, we show that Hilbert transforms along some curves are bounded on $L^{p}\left(\mathbb{R}^{n} ; X\right)$ for some $1<p<\infty$ and some UMD spaces $X$. In particular, we prove that Hilbert transforms along some curves are completely $L^{p}$-bounded in the terminology from operator space theory. Moreover, we obtain the $L^{p}\left(\mathbb{R}^{n} ; X\right)$-boundedness of anisotropic singular integrals by using the "method of rotations" of Calderón-Zygmund. All these results extend preexisting related ones.
\end{abstract}

\section{INTRODUCTION}

The question of whether the mapping properties of singular integral operators could be extended to the Lebesgue-Bôhner spaces $L^{p}\left(\mathbb{R}^{n} ; X\right)(1<p<\infty)$ of vector-valued functions was taken up by several authors in the 60s. In [1], Benedek, Calderón, and Panzone observed that the boundedness on $L^{p_{0}}\left(\mathbb{R}^{n} ; X\right)$ for one $1<p_{0}<\infty$ of a singular integral operator, together with Hörmander's condition, implies its boundedness on $L^{p}\left(\mathbb{R}^{n} ; X\right)$ for all $1<p<\infty$. However, to actually get the $L^{p_{0}}\left(\mathbb{R}^{n} ; X\right)$-boundedness (something that was immediate for $p_{0}=2$ in the scalar-valued) turned out to be a significantly difficult task except in the case $X=L^{p_{0}}(\Omega)$ for some measure space $\Omega$.

The first progress made in this direction is Burkholder's [3] extension of Riesz's classical theorem on the $L^{p}$-boundedness of the Hilbert transform, where it was shown that if the underlying Banach space $X$ satisfies the so-called UMD-property,

Copyright 2016 by the Tusi Mathematical Research Group.

Received Apr. 9, 2015; Accepted Jul. 27, 2015.

${ }^{*}$ Corresponding author.

2010 Mathematics Subject Classification. Primary 43A32; Secondary 46 B99.

Keywords. Hilbert transforms along curves, weighted Hörmander condition, UMD spaces, completely bounded, analytic interpolation. 


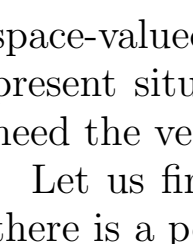

Banach J. Math. Anal. 10 (2016), no. 2, 430-450

http://dx.doi.org/10.1215/17358787-3589397

ISSN: $1735-8787$ (electronic)

http://projecteuclid.org/bjma

\title{
VECTOR-VALUED HILBERT TRANSFORMS ALONG CURVES
}

\author{
GUIXIANG HONG ${ }^{1}$ and HONGHAI LIU ${ }^{2 *}$ \\ Communicated by Q. Xu
}

\begin{abstract}
In this paper, we show that Hilbert transforms along some curves are bounded on $L^{p}\left(\mathbb{R}^{n} ; X\right)$ for some $1<p<\infty$ and some UMD spaces $X$. In particular, we prove that Hilbert transforms along some curves are completely $L^{p}$-bounded in the terminology from operator space theory. Moreover, we obtain the $L^{p}\left(\mathbb{R}^{n} ; X\right)$-boundedness of anisotropic singular integrals by using the "method of rotations" of Calderón-Zygmund. All these results extend preexisting related ones.
\end{abstract}

\section{INTRODUCTION}

The question of whether the mapping properties of singular integral operators could be extended to the Lebesgue-Bôhner spaces $L^{p}\left(\mathbb{R}^{n} ; X\right)(1<p<\infty)$ of vector-valued functions was taken up by several authors in the 60s. In [1], Benedek, Calderón, and Panzone observed that the boundedness on $L^{p_{0}}\left(\mathbb{R}^{n} ; X\right)$ for one $1<p_{0}<\infty$ of a singular integral operator, together with Hörmander's condition, implies its boundedness on $L^{p}\left(\mathbb{R}^{n} ; X\right)$ for all $1<p<\infty$. However, to actually get the $L^{p_{0}}\left(\mathbb{R}^{n} ; X\right)$-boundedness (something that was immediate for $p_{0}=2$ in the scalar-valued) turned out to be a significantly difficult task except in the case $X=L^{p_{0}}(\Omega)$ for some measure space $\Omega$.

The first progress made in this direction is Burkholder's [3] extension of Riesz's classical theorem on the $L^{p}$-boundedness of the Hilbert transform, where it was shown that if the underlying Banach space $X$ satisfies the so-called UMD-property,

Copyright 2016 by the Tusi Mathematical Research Group.

Received Apr. 9, 2015; Accepted Jul. 27, 2015.

${ }^{*}$ Corresponding author.

2010 Mathematics Subject Classification. Primary 43A32; Secondary 46 B99.

Keywords. Hilbert transforms along curves, weighted Hörmander condition, UMD spaces, completely bounded, analytic interpolation. 


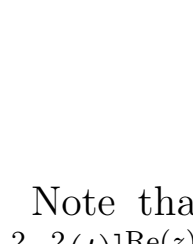

Banach J. Math. Anal. 10 (2016), no. 2, 430-450

http://dx.doi.org/10.1215/17358787-3589397

ISSN: $1735-8787$ (electronic)

http://projecteuclid.org/bjma

\title{
VECTOR-VALUED HILBERT TRANSFORMS ALONG CURVES
}

\author{
GUIXIANG HONG ${ }^{1}$ and HONGHAI LIU ${ }^{2 *}$ \\ Communicated by Q. Xu
}

\begin{abstract}
In this paper, we show that Hilbert transforms along some curves are bounded on $L^{p}\left(\mathbb{R}^{n} ; X\right)$ for some $1<p<\infty$ and some UMD spaces $X$. In particular, we prove that Hilbert transforms along some curves are completely $L^{p}$-bounded in the terminology from operator space theory. Moreover, we obtain the $L^{p}\left(\mathbb{R}^{n} ; X\right)$-boundedness of anisotropic singular integrals by using the "method of rotations" of Calderón-Zygmund. All these results extend preexisting related ones.
\end{abstract}

\section{INTRODUCTION}

The question of whether the mapping properties of singular integral operators could be extended to the Lebesgue-Bôhner spaces $L^{p}\left(\mathbb{R}^{n} ; X\right)(1<p<\infty)$ of vector-valued functions was taken up by several authors in the 60s. In [1], Benedek, Calderón, and Panzone observed that the boundedness on $L^{p_{0}}\left(\mathbb{R}^{n} ; X\right)$ for one $1<p_{0}<\infty$ of a singular integral operator, together with Hörmander's condition, implies its boundedness on $L^{p}\left(\mathbb{R}^{n} ; X\right)$ for all $1<p<\infty$. However, to actually get the $L^{p_{0}}\left(\mathbb{R}^{n} ; X\right)$-boundedness (something that was immediate for $p_{0}=2$ in the scalar-valued) turned out to be a significantly difficult task except in the case $X=L^{p_{0}}(\Omega)$ for some measure space $\Omega$.

The first progress made in this direction is Burkholder's [3] extension of Riesz's classical theorem on the $L^{p}$-boundedness of the Hilbert transform, where it was shown that if the underlying Banach space $X$ satisfies the so-called UMD-property,

Copyright 2016 by the Tusi Mathematical Research Group.

Received Apr. 9, 2015; Accepted Jul. 27, 2015.

${ }^{*}$ Corresponding author.

2010 Mathematics Subject Classification. Primary 43A32; Secondary 46 B99.

Keywords. Hilbert transforms along curves, weighted Hörmander condition, UMD spaces, completely bounded, analytic interpolation. 


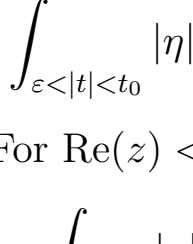

Banach J. Math. Anal. 10 (2016), no. 2, 430-450

http://dx.doi.org/10.1215/17358787-3589397

ISSN: $1735-8787$ (electronic)

http://projecteuclid.org/bjma

\title{
VECTOR-VALUED HILBERT TRANSFORMS ALONG CURVES
}

\author{
GUIXIANG HONG ${ }^{1}$ and HONGHAI LIU ${ }^{2 *}$ \\ Communicated by Q. Xu
}

\begin{abstract}
In this paper, we show that Hilbert transforms along some curves are bounded on $L^{p}\left(\mathbb{R}^{n} ; X\right)$ for some $1<p<\infty$ and some UMD spaces $X$. In particular, we prove that Hilbert transforms along some curves are completely $L^{p}$-bounded in the terminology from operator space theory. Moreover, we obtain the $L^{p}\left(\mathbb{R}^{n} ; X\right)$-boundedness of anisotropic singular integrals by using the "method of rotations" of Calderón-Zygmund. All these results extend preexisting related ones.
\end{abstract}

\section{INTRODUCTION}

The question of whether the mapping properties of singular integral operators could be extended to the Lebesgue-Bôhner spaces $L^{p}\left(\mathbb{R}^{n} ; X\right)(1<p<\infty)$ of vector-valued functions was taken up by several authors in the 60s. In [1], Benedek, Calderón, and Panzone observed that the boundedness on $L^{p_{0}}\left(\mathbb{R}^{n} ; X\right)$ for one $1<p_{0}<\infty$ of a singular integral operator, together with Hörmander's condition, implies its boundedness on $L^{p}\left(\mathbb{R}^{n} ; X\right)$ for all $1<p<\infty$. However, to actually get the $L^{p_{0}}\left(\mathbb{R}^{n} ; X\right)$-boundedness (something that was immediate for $p_{0}=2$ in the scalar-valued) turned out to be a significantly difficult task except in the case $X=L^{p_{0}}(\Omega)$ for some measure space $\Omega$.

The first progress made in this direction is Burkholder's [3] extension of Riesz's classical theorem on the $L^{p}$-boundedness of the Hilbert transform, where it was shown that if the underlying Banach space $X$ satisfies the so-called UMD-property,

Copyright 2016 by the Tusi Mathematical Research Group.

Received Apr. 9, 2015; Accepted Jul. 27, 2015.

${ }^{*}$ Corresponding author.

2010 Mathematics Subject Classification. Primary 43A32; Secondary 46 B99.

Keywords. Hilbert transforms along curves, weighted Hörmander condition, UMD spaces, completely bounded, analytic interpolation. 


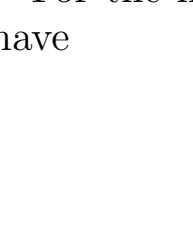

Banach J. Math. Anal. 10 (2016), no. 2, 430-450

http://dx.doi.org/10.1215/17358787-3589397

ISSN: $1735-8787$ (electronic)

http://projecteuclid.org/bjma

\title{
VECTOR-VALUED HILBERT TRANSFORMS ALONG CURVES
}

\author{
GUIXIANG HONG ${ }^{1}$ and HONGHAI LIU ${ }^{2 *}$ \\ Communicated by Q. Xu
}

\begin{abstract}
In this paper, we show that Hilbert transforms along some curves are bounded on $L^{p}\left(\mathbb{R}^{n} ; X\right)$ for some $1<p<\infty$ and some UMD spaces $X$. In particular, we prove that Hilbert transforms along some curves are completely $L^{p}$-bounded in the terminology from operator space theory. Moreover, we obtain the $L^{p}\left(\mathbb{R}^{n} ; X\right)$-boundedness of anisotropic singular integrals by using the "method of rotations" of Calderón-Zygmund. All these results extend preexisting related ones.
\end{abstract}

\section{INTRODUCTION}

The question of whether the mapping properties of singular integral operators could be extended to the Lebesgue-Bôhner spaces $L^{p}\left(\mathbb{R}^{n} ; X\right)(1<p<\infty)$ of vector-valued functions was taken up by several authors in the 60s. In [1], Benedek, Calderón, and Panzone observed that the boundedness on $L^{p_{0}}\left(\mathbb{R}^{n} ; X\right)$ for one $1<p_{0}<\infty$ of a singular integral operator, together with Hörmander's condition, implies its boundedness on $L^{p}\left(\mathbb{R}^{n} ; X\right)$ for all $1<p<\infty$. However, to actually get the $L^{p_{0}}\left(\mathbb{R}^{n} ; X\right)$-boundedness (something that was immediate for $p_{0}=2$ in the scalar-valued) turned out to be a significantly difficult task except in the case $X=L^{p_{0}}(\Omega)$ for some measure space $\Omega$.

The first progress made in this direction is Burkholder's [3] extension of Riesz's classical theorem on the $L^{p}$-boundedness of the Hilbert transform, where it was shown that if the underlying Banach space $X$ satisfies the so-called UMD-property,

Copyright 2016 by the Tusi Mathematical Research Group.

Received Apr. 9, 2015; Accepted Jul. 27, 2015.

${ }^{*}$ Corresponding author.

2010 Mathematics Subject Classification. Primary 43A32; Secondary 46 B99.

Keywords. Hilbert transforms along curves, weighted Hörmander condition, UMD spaces, completely bounded, analytic interpolation. 


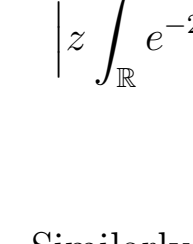

Banach J. Math. Anal. 10 (2016), no. 2, 430-450

http://dx.doi.org/10.1215/17358787-3589397

ISSN: $1735-8787$ (electronic)

http://projecteuclid.org/bjma

\title{
VECTOR-VALUED HILBERT TRANSFORMS ALONG CURVES
}

\author{
GUIXIANG HONG ${ }^{1}$ and HONGHAI LIU ${ }^{2 *}$ \\ Communicated by Q. Xu
}

\begin{abstract}
In this paper, we show that Hilbert transforms along some curves are bounded on $L^{p}\left(\mathbb{R}^{n} ; X\right)$ for some $1<p<\infty$ and some UMD spaces $X$. In particular, we prove that Hilbert transforms along some curves are completely $L^{p}$-bounded in the terminology from operator space theory. Moreover, we obtain the $L^{p}\left(\mathbb{R}^{n} ; X\right)$-boundedness of anisotropic singular integrals by using the "method of rotations" of Calderón-Zygmund. All these results extend preexisting related ones.
\end{abstract}

\section{INTRODUCTION}

The question of whether the mapping properties of singular integral operators could be extended to the Lebesgue-Bôhner spaces $L^{p}\left(\mathbb{R}^{n} ; X\right)(1<p<\infty)$ of vector-valued functions was taken up by several authors in the 60s. In [1], Benedek, Calderón, and Panzone observed that the boundedness on $L^{p_{0}}\left(\mathbb{R}^{n} ; X\right)$ for one $1<p_{0}<\infty$ of a singular integral operator, together with Hörmander's condition, implies its boundedness on $L^{p}\left(\mathbb{R}^{n} ; X\right)$ for all $1<p<\infty$. However, to actually get the $L^{p_{0}}\left(\mathbb{R}^{n} ; X\right)$-boundedness (something that was immediate for $p_{0}=2$ in the scalar-valued) turned out to be a significantly difficult task except in the case $X=L^{p_{0}}(\Omega)$ for some measure space $\Omega$.

The first progress made in this direction is Burkholder's [3] extension of Riesz's classical theorem on the $L^{p}$-boundedness of the Hilbert transform, where it was shown that if the underlying Banach space $X$ satisfies the so-called UMD-property,

Copyright 2016 by the Tusi Mathematical Research Group.

Received Apr. 9, 2015; Accepted Jul. 27, 2015.

${ }^{*}$ Corresponding author.

2010 Mathematics Subject Classification. Primary 43A32; Secondary 46 B99.

Keywords. Hilbert transforms along curves, weighted Hörmander condition, UMD spaces, completely bounded, analytic interpolation. 


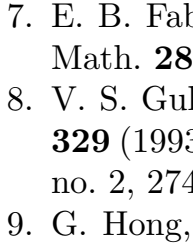

Banach J. Math. Anal. 10 (2016), no. 2, 430-450

http://dx.doi.org/10.1215/17358787-3589397

ISSN: $1735-8787$ (electronic)

http://projecteuclid.org/bjma

\title{
VECTOR-VALUED HILBERT TRANSFORMS ALONG CURVES
}

\author{
GUIXIANG HONG ${ }^{1}$ and HONGHAI LIU ${ }^{2 *}$ \\ Communicated by Q. Xu
}

\begin{abstract}
In this paper, we show that Hilbert transforms along some curves are bounded on $L^{p}\left(\mathbb{R}^{n} ; X\right)$ for some $1<p<\infty$ and some UMD spaces $X$. In particular, we prove that Hilbert transforms along some curves are completely $L^{p}$-bounded in the terminology from operator space theory. Moreover, we obtain the $L^{p}\left(\mathbb{R}^{n} ; X\right)$-boundedness of anisotropic singular integrals by using the "method of rotations" of Calderón-Zygmund. All these results extend preexisting related ones.
\end{abstract}

\section{INTRODUCTION}

The question of whether the mapping properties of singular integral operators could be extended to the Lebesgue-Bôhner spaces $L^{p}\left(\mathbb{R}^{n} ; X\right)(1<p<\infty)$ of vector-valued functions was taken up by several authors in the 60s. In [1], Benedek, Calderón, and Panzone observed that the boundedness on $L^{p_{0}}\left(\mathbb{R}^{n} ; X\right)$ for one $1<p_{0}<\infty$ of a singular integral operator, together with Hörmander's condition, implies its boundedness on $L^{p}\left(\mathbb{R}^{n} ; X\right)$ for all $1<p<\infty$. However, to actually get the $L^{p_{0}}\left(\mathbb{R}^{n} ; X\right)$-boundedness (something that was immediate for $p_{0}=2$ in the scalar-valued) turned out to be a significantly difficult task except in the case $X=L^{p_{0}}(\Omega)$ for some measure space $\Omega$.

The first progress made in this direction is Burkholder's [3] extension of Riesz's classical theorem on the $L^{p}$-boundedness of the Hilbert transform, where it was shown that if the underlying Banach space $X$ satisfies the so-called UMD-property,

Copyright 2016 by the Tusi Mathematical Research Group.

Received Apr. 9, 2015; Accepted Jul. 27, 2015.

${ }^{*}$ Corresponding author.

2010 Mathematics Subject Classification. Primary 43A32; Secondary 46 B99.

Keywords. Hilbert transforms along curves, weighted Hörmander condition, UMD spaces, completely bounded, analytic interpolation. 


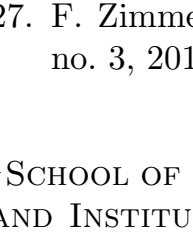

Banach J. Math. Anal. 10 (2016), no. 2, 430-450

http://dx.doi.org/10.1215/17358787-3589397

ISSN: $1735-8787$ (electronic)

http://projecteuclid.org/bjma

\title{
VECTOR-VALUED HILBERT TRANSFORMS ALONG CURVES
}

\author{
GUIXIANG HONG ${ }^{1}$ and HONGHAI LIU ${ }^{2 *}$ \\ Communicated by Q. Xu
}

\begin{abstract}
In this paper, we show that Hilbert transforms along some curves are bounded on $L^{p}\left(\mathbb{R}^{n} ; X\right)$ for some $1<p<\infty$ and some UMD spaces $X$. In particular, we prove that Hilbert transforms along some curves are completely $L^{p}$-bounded in the terminology from operator space theory. Moreover, we obtain the $L^{p}\left(\mathbb{R}^{n} ; X\right)$-boundedness of anisotropic singular integrals by using the "method of rotations" of Calderón-Zygmund. All these results extend preexisting related ones.
\end{abstract}

\section{INTRODUCTION}

The question of whether the mapping properties of singular integral operators could be extended to the Lebesgue-Bôhner spaces $L^{p}\left(\mathbb{R}^{n} ; X\right)(1<p<\infty)$ of vector-valued functions was taken up by several authors in the 60s. In [1], Benedek, Calderón, and Panzone observed that the boundedness on $L^{p_{0}}\left(\mathbb{R}^{n} ; X\right)$ for one $1<p_{0}<\infty$ of a singular integral operator, together with Hörmander's condition, implies its boundedness on $L^{p}\left(\mathbb{R}^{n} ; X\right)$ for all $1<p<\infty$. However, to actually get the $L^{p_{0}}\left(\mathbb{R}^{n} ; X\right)$-boundedness (something that was immediate for $p_{0}=2$ in the scalar-valued) turned out to be a significantly difficult task except in the case $X=L^{p_{0}}(\Omega)$ for some measure space $\Omega$.

The first progress made in this direction is Burkholder's [3] extension of Riesz's classical theorem on the $L^{p}$-boundedness of the Hilbert transform, where it was shown that if the underlying Banach space $X$ satisfies the so-called UMD-property,

Copyright 2016 by the Tusi Mathematical Research Group.

Received Apr. 9, 2015; Accepted Jul. 27, 2015.

${ }^{*}$ Corresponding author.

2010 Mathematics Subject Classification. Primary 43A32; Secondary 46 B99.

Keywords. Hilbert transforms along curves, weighted Hörmander condition, UMD spaces, completely bounded, analytic interpolation. 